
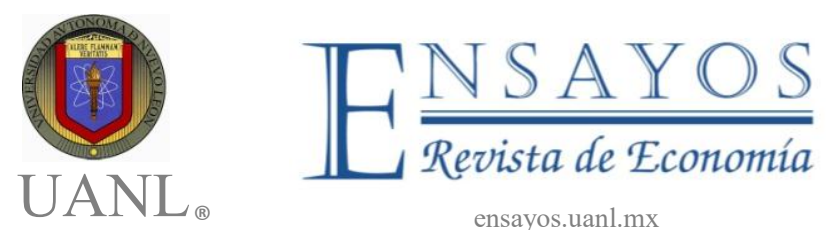

ensayos.uanl.mx

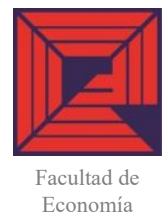

\title{
Salario eficiente y crecimiento económico para el caso de América Latina
}

\author{
Efficient wages and Economic Growth in Latin America
}
Tomás Gómez Rodríguez* Humberto Ríos Bolívar Ali Aali Bujari

\begin{tabular}{|c|c|}
\hline $\begin{array}{l}\text { Información del } \\
\text { artículo }\end{array}$ & Resumen \\
\hline $\begin{array}{l}\text { Recibido: } \\
9 \text { septiembre } 2017 \\
\text { Aceptado: } \\
21 \text { septiembre } 2018\end{array}$ & $\begin{array}{l}\text { En la actualidad, la investigación sobre el salario } \\
\text { eficiente ha llamado la atención acerca de la } \\
\text { posibilidad de que incrementos salariales puedan } \\
\text { aumentar la productividad y, por lo tanto, el }\end{array}$ \\
\hline $\begin{array}{l}\text { Clasificación JEL: } \\
\text { E24 ; F43 ; J24 ; J30 } \\
\text { Palabras clave: } \\
\text { Crecimiento } \\
\text { Económico; Salarios } \\
\text { Eficientes; Eficiencia } \\
\text { Técnica }\end{array}$ & $\begin{array}{l}\text { plantea la hipótesis de que el desempleo y la } \\
\text { inflación sean variables que aumentan la } \\
\text { ineficiencia. Para estudiar estos posibles } \\
\text { resultados, se utiliza un enfoque de frontera de } \\
\text { producción estocástica para panel de datos, en un } \\
\text { período que abarca del año } 1995 \text { al año } 2011 \text {. Los } \\
\text { países considerados en el estudio son: Argentina, } \\
\text { Brasil, Colombia, Chile, México, Perú y } \\
\text { Venezuela. Se encuentra evidencia de que existe } \\
\text { una relación positiva entre aumento salarial y } \\
\text { crecimiento económico. Así mismo, los resultados } \\
\text { muestran que el desempleo y la inflación son } \\
\text { variables que aumentan los efectos de } \\
\text { ineficiencia. }\end{array}$ \\
\hline
\end{tabular}

\footnotetext{
*Universidad Autónoma del Estado de Hidalgo. Correo electrónico: yhwh13@gmail.com

** Escuela Superior de Economía del Instituto Politécnico Nacional

${ }^{* * *}$ Universidad Autónoma del Estado de Hidalgo
} 


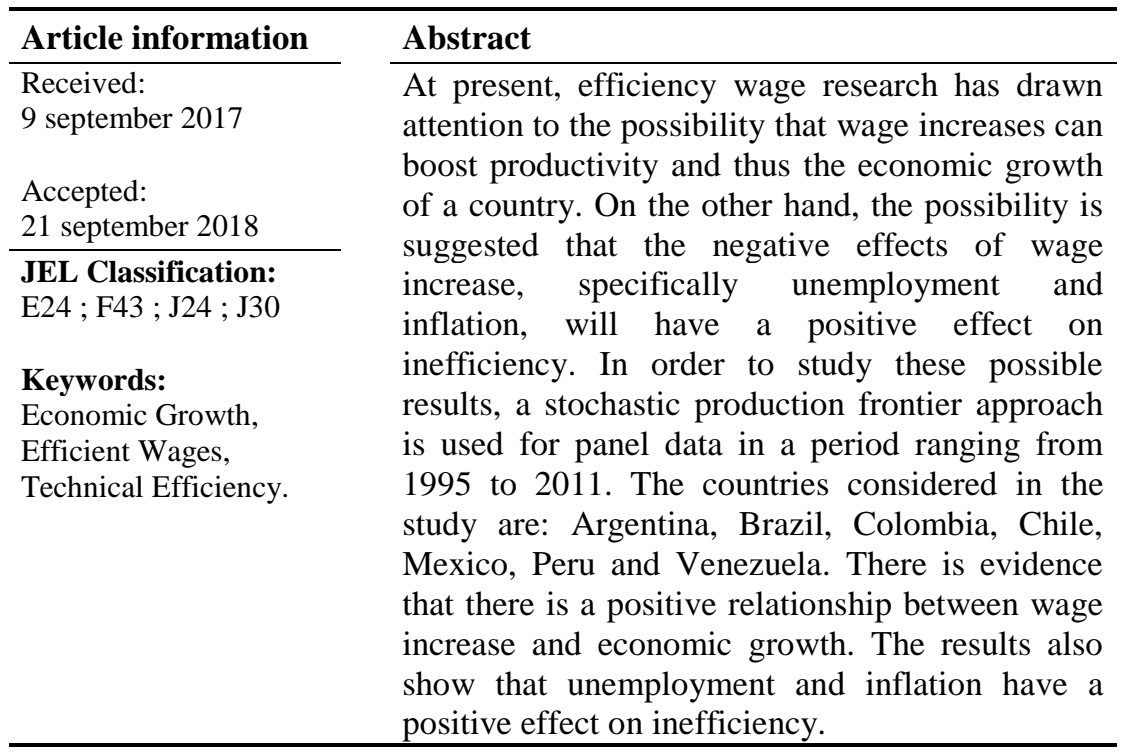

\section{Introducción}

Los salarios son la principal fuente de ingreso para los trabajadores y tienen un efecto decisivo en los niveles de vida de ellos y de sus familias. La relación entre aumento salarial y crecimiento económico se ha vuelto un tema de particular importancia en años recientes, a la luz de la actual crisis económica que se desató en 2008. El nexo entre salarios y crecimiento económico también juega un papel importante en el progresivo aumento de las desigualdades entre los países, y en la creciente necesidad de encontrar nuevas fórmulas de estimular el crecimiento. Así mismo, el nexo entre salarios y crecimiento económico es importante porque los salarios son un determinante clave de los estándares de vida de la población empleada, como lo es también la distribución del ingreso entre capital y trabajo.

Sin embargo, esta posible relación ha sido una fuente substancial de controversias no solo por su importancia intrínseca, sino también por los problemas conceptuales de medición que se originan al hacer comparaciones (Feldstein, 2008). Los modelos de Shapiro y Stiglitz (1984) y Solow (1979) argumentan que salarios más altos conducen a un mayor esfuerzo por parte del trabajador y, en consecuencia, aumentan la producción de la firma o industria. Por lo tanto, la hipótesis del salario eficiente es particularmente importante para países en desarrollo, dado que, si se demuestra su validez, plantearía importantes preguntas acerca de la efectividad de las políticas de estabilización y ajuste estructural. Por lo cual, se estudia la relación entre salarios y crecimiento económico a través de la hipótesis del salario eficiente. 
Por otra parte, el análisis del nexo salario-crecimiento económico se torna más interesante cuando se hace a través de la medición de la eficiencia técnica, ya que la productividad general de un sistema económico está directamente relacionada con la eficiencia en la utilización de los componentes, con los cuales se lleva a cabo la producción dentro del sistema. Este trabajo se apoya, para el análisis de la relación salario-crecimiento, en la hipótesis de eficiencia salarial, específicamente, se utiliza un modelo de crecimiento económico endógeno modificado para incluir la hipótesis del salario eficiente ${ }^{1}$ propuesto por Choi (1995); este modelo está basado en el trabajo de Goodwin (1967), el cual asume una economía de un sector, no lineal y no monetaria. El modelo plantea que, con una tecnología de producción de coeficientes fijos, una tasa elevada de acumulación de capital provoca un aumento en el empleo. El crecimiento en el empleo gradualmente fortalece el poder de negociación de los trabajadores en el mercado de trabajo. Y, cuando la economía se acerca a la barrera natural de pleno empleo, provoca que de manera eventual aumente la tasa del salario real, cambiando la distribución del ingreso a favor del trabajo. En la hipótesis del salario eficiente, está implícito el supuesto de que al pagar salarios más altos ex ante, resulta en un aumento de productividad ex post, al motivar a los trabajadores para realizar un mayor esfuerzo o al atraer trabajadores de mayor calidad. La conjetura de que los aumentos en el salario se pagan por sí mismos a través de una mayor productividad, está explícita en la hipótesis.

Los supuestos e implicaciones de la hipótesis de salario eficiente han sido extensamente investigados en la literatura económica, sociológica y contable, sin embargo, han sido relativamente pocos los esfuerzos para probar empíricamente la relación entre salarios y el desempeño real de la economía con datos reales. Por lo cual, se realiza el análisis de la relación salariocrecimiento usando datos de siete países de América Latina, en un período que abarca del año 1995 al año 2011. Los países seleccionados son: Argentina, Brasil, Colombia, Chile, México, Perú y Venezuela. En esta investigación se abordan dos preguntas fundamentales. La primer pregunta es: ¿salarios más altos llevan a un mejor desempeño económico?; y la segunda, ¿la inflación y el desempleo son variables que afectan la ineficiencia?

De las dos preguntas centrales de esta investigación surgen dos hipótesis: la primera supone que un aumento en el nivel salarial permite alcanzar mayor crecimiento económico; la segunda, que el desempleo y la inflación son variables que aumentan la ineficiencia. Por lo que el objetivo de esta investigación es analizar la relación entre salarios y crecimiento económico,

\footnotetext{
${ }^{1}$ Para la formulación de la hipótesis del salario eficiente, se refiere a Weiss (1990)
} 
por una parte, y por otra, analizar los efectos negativos de la inflación y el desempleo, usando el método de frontera de producción para panel de datos. Para alcanzar dichos objetivos, se recurre específicamente al método de frontera de producción estocástica para panel de datos, presentado por Battese y Coelli (1995), el cual permite, por una parte, estimar la productividad de los factores de producción y por otra, determinar los efectos de ineficiencia de las variables inflación y desempleo. Se usa esta técnica de frontera de producción, ya que los métodos de dos etapas son conocidos por tener dos tipos de sesgos: el primero se da cuando el conjunto de insumos y las variables exógenas se encuentran correlacionados, y el segundo, cuando las medidas de eficiencia técnica en la primer etapa probablemente estén poco dispersas, de modo que los resultados de la regresión en la segunda etapa estén sesgadas a la baja (Wang y Schmidt, 2002).

Este trabajo de investigación se divide en las siguientes secciones: Revisión de la literatura, en este apartado se incluyen los diferentes enfoques bajo los cuales se estudia la relación salario-crecimiento; Marco teórico, en esta sección se incluye la fundamentación teórica de la relación salariocrecimiento. El resto de las secciones son: Especificación del modelo, Datos, Resultados y Conclusiones.

\section{Revisión de la literatura}

En la literatura, se distinguen dos vertientes en el estudio de la relación salario-crecimiento. Por un lado, se encuentran modelos macroeconómicos agregados; mientras que, por otro lado, existen trabajos que a partir de la hipótesis del salario eficiente analizan la relación salario-crecimiento.

Existe un tema importante en la literatura que ha sido largamente debatido, este tema consiste en evaluar los efectos del salario mínimo en los modelos macroeconómicos agregados. Los que se oponen ven al salario mínimo como una mala política social, esencialmente porque deteriora el empleo y la producción. Mientras que los defensores, por otro lado, típicamente se concentran en metas redistributivas que el salario mínimo puede crear.

Uno de los primeros trabajos que analiza los efectos del salario mínimo en modelos macroeconómicos agregados, es el de Stigler (1946). En este trabajo, se propone un modelo básico para medir los efectos del salario mínimo en el empleo y desempleo, centrado en un mercado laboral con trabajadores homogéneos, en el cual se predice que el salario mínimo causa reducciones en el producto y en el empleo. A continuación, se presenta una breve revisión de algunas de las contribuciones relacionadas con el salario mínimo, desempleo y crecimiento en el nivel macroeconómico. 
Al estudiar cómo el salario mínimo para trabajadores no calificados afecta al crecimiento económico y al bienestar, en un modelo de generaciones superpuestas y una economía cerrada de dos sectores con crecimiento endógeno del tipo Lucas (1988), Cahuc y Michel (1996) encuentran que cuando se introduce un salario mínimo vinculante en el mercado de trabajadores no calificados, este tiene un efecto positivo externo en la acumulación de capital humano, porque la demanda por trabajadores calificados aumenta y, por lo tanto, los trabajadores no calificados intentarán mejorar su nivel de habilidades para poder evitar el desempleo. Debido a esta externalidad, el salario mínimo puede promover crecimiento y bienestar. No obstante, los autores resaltan que, en un contexto exógeno, el salario mínimo incrementa el desempleo y reduce el crecimiento. Un trabajo muy relacionado con el de Cahuc y Michel (1996), es el trabajo de Ravn y Sorensen (1999), quienes encuentran que un aumento del salario mínimo tiene un efecto incierto en el crecimiento de la producción.

Usando un modelo de crecimiento endógeno en una economía abierta, Askenazy (2003) estudia el impacto del salario mínimo sobre el crecimiento de un país. Encuentra que el crecimiento a largo plazo se acelera en proporción a las exportaciones. Para alcanzar este resultado, Askenazy (2003) emplea una muestra de once países de la OCDE.

En el trabajo de Fanti y Gori (2011), se analiza la relación positiva entre salarios mínimos y crecimiento económico en una economía basada en el modelo de Romer (1986) de un solo sector con generaciones superpuestas; si se asume la existencia de beneficios de desempleo, los cuales son financiados con un presupuesto balanceado. Fanti y Gori (2011) demuestran que el salario mínimo puede promover el crecimiento económico y el bienestar aún con la existencia de desempleo.

El trabajo de Sabia (2015) analiza la hipótesis de que el salario mínimo podría ser un motor del crecimiento, y sus hallazgos demuestran que el salario mínimo puede incrementar el crecimiento económico en el nivel macroeconómico, esto si la productividad cambia hacia sectores con mayores habilidades, posiblemente porque se promueve una mayor capacitación en los trabajadores con menos habilidades.

Basado en la simulación y un modelo de crecimiento económico con múltiples agentes, $\mathrm{Xu}$, Huo y Shang (2015) diseñan un mecanismo para simular el impacto de la distribución del ingreso en los ciclos económicos. En el análisis empírico, los autores utilizan datos de los Estados Unidos de entre 1982 y 2003. Sus resultados muestran que existe una relación inherente entre los ciclos de crecimiento económico y la proporción de salario mínimo a salario medio. 
Finalmente, Yamaguchi (2017) encuentra que el salario mínimo es una política efectiva para superar la deflación y la estagnación, aunque incrementa la tasa natural de desempleo. En su investigación, el autor emplea un modelo dinámico de equilibrio general sin fricciones de mercado.

La otra perspectiva en la literatura se enfoca en modelos de eficiencia salarial, los cuales se han empleado para analizar diferentes relaciones como: salario-desempleo, salario-crecimiento, inflación-crecimiento y desempleocrecimiento. Los trabajos que incorporan la hipótesis del salario eficiente se dividen en tres clases: la primera comprende trabajos que emplean funciones de producción aumentadas para analizar la relación salario-productividad; la segunda, trabajos que examinan la interrelación entre salario, productividad e inflación, que utilizan principalmente series de tiempo y pruebas de causalidad; por último, la tercera clase incluye trabajos que emplean fronteras de producción estocástica, los cuales son utilizados para analizar la relación salario-crecimiento.

En la primera clasificación se encuentran estudios que investigan la relación entre salarios y productividad, mediante el aumento de una función de producción, con medidas del salario relativo promedio para una empresa o industria. En un estudio con 219 firmas manufactureras, Wadhwani y Wall (1991) realizan una regresión usando los cambios en las ventas en relación con los cambios en el salario y un conjunto de variables relacionadas con la producción, y encontraron que los cambios en las ventas están positivamente correlacionados con los cambios relativos en el salario. Levine (1992) obteniene resultados similares con una muestra de 369 unidades.

Usando el teorema de bifurcación Hopf, Choi (1995) examina la robustez del modelo de Goodwin (1967), cuando el nivel de esfuerzo de los trabajadores depende de los salarios reales. Sus resultados demuestran que si el nivel de esfuerzo disminuye, a medida que aumenta el salario real, el modelo da origen a un ciclo de crecimiento.

El trabajo de Meckl (2001) examina las implicaciones del desempleo, inducido por los salarios eficientes dentro de un modelo de crecimiento endógeno, y encuentra que existe una correlación entre la tasa de crecimiento y la tasa de desempleo. El signo de esta correlación está determinado por el diferencial salarial intersectorial.

Huang, Hallam, Orazem y Paterno (1998) utilizan diferentes funciones de producción para probar las proposiciones de la hipótesis del salario eficiente, para el sector manufacturero en el nivel industrial; sus resultados demuestran que el aumento salarial y el alto desempleo aumentan la productividad. Específicamente, encuentran que el $88 \%$ del efecto de la productividad 
asociado con los salarios industriales, dependen del capital humano, y solo el $12 \%$ depende del aumento salarial.

Mertzanis (2009) utiliza la hipótesis del salario eficiente y una función de esfuerzo, donde la productividad del trabajo depende de la distribución del ingreso entre salario, ganancias y el nivel general de producción. Esta función se incorpora en un modelo de crecimiento económico estructuralista keynesiano, en el cual, las decisiones de inversión dependen de la distribución del ingreso, la inflación y el nivel de producción. La dirección y magnitud del impacto general sobre la inflación y el crecimiento dependen de factores institucionales, como: la especificación de la función de esfuerzo, las diferentes propensiones al ahorro, los determinantes de la acumulación de capital y el estado de la distribución del ingreso.

Zagler (2011) emplea un modelo con un sector manufacturero que exhibe competencia monopolística y un sector que compite en innovación, ambos pagan salarios de eficiencia. Se establece que existe una relación causal entre la tasa de crecimiento y la tasa de desempleo siempre y cuando existan diferentes niveles de eficiencia entre los sectores.

Partiendo de una confrontación entre teoría microeconómica y datos macroeconómicos, Razzak (2015) establece que la mayoría de las teorías de salarios eficientes y negociación predicen que, durante el ciclo de negocios, la tasa de desempleo cae por debajo de su tasa natural, cuando el salario real del trabajador excede el salario de reserva. Razzak encuentra que tomar en cuenta las decisiones en el nivel microeconómico ayuda a explicar casi todas las fluctuaciones del desempleo en los Estados Unidos.

Tadjoeddin (2016) examina la relación entre productividad, salarios y empleo en el sector manufacturero de Indonesia. Se desagregan salarios y productividad para empresas grandes y pequeñas. La investigación apunta que, cuando se considera el sector manufacturero en general, se observa una divergencia entre el salario real y la productividad. Pero cuando se examinan solo a las empresas grandes, entonces se encuentra que existe una correlación positiva entre salario y productividad.

La segunda clase de trabajos incluyen estudios que investigan la interrelación entre productividad, salarios reales y desempleo. Este tipo de trabajos se caracteriza por el uso de pruebas de cointegración y causalidad.

Kumar, Webber y Perry (2012) estudian la interrelación entre salario real, productividad e inflación para el caso de Australia. Se utilizan pruebas de cointegración, causalidad y de cambio estructural, las cuales se aplican a los datos del período 1965 a 2007. Se corrobora la presencia de un cambio 
estructural en 1985, y en él se muestra que un $1 \%$ de aumento en el salario real del sector manufacturero lleva a un incremento de entre $.5 \%$ y $.8 \%$, en la productividad del sector. Así mismo, los resultados de la prueba de Granger sugieren que la dirección de la causalidad va desde los salarios reales y la inflación a la productividad del trabajo, en el largo plazo.

Sobre el mismo tema, Tang (2014) prueba la interrelación entre salario real, productividad e inflación para el caso de Malasia, mediante el enfoque de límites para la prueba de cointegración y la prueba de causalidad de Granger. Sus hallazgos sugieren que la inflación está relacionada negativamente con la productividad del trabajo; mientras que la relación entre salario real y productividad del trabajo es no lineal, con forma de "u" invertida. La prueba de causalidad de Granger muestra que los salarios reales causan la productividad del trabajo, pero no se encuentra evidencia de causalidad reversa.

Teniendo como objetivo examinar las interrelaciones entre productividad, salarios reales e inflación en el sector manufacturero de Turquía, Yildirim (2015) utiliza un análisis de cointegración y la prueba de causalidad de Granger, para el período 1998-2002. Se concluye que existe una fuerte retroalimentación entre productividad del trabajo e inflación. Examinando la relación de largo y corto plazo entre salario real, inflación y productividad del trabajo en Nigeria, para el período 1981-2012.

Iheanacho (2017) emplea un enfoque autorregresivo de atrasos distribuidos y un enfoque de prueba y error para el análisis de cointegración. La evidencia obtenida sugiere que existe una relación de largo plazo entre salario real y productividad del trabajo, así mismo, se encuentra evidencia de la existencia de una relación positiva entre inflación y productividad del trabajo.

La tercera vertiente de la literatura son los estudios que utilizan fronteras de producción para tratar de probar la hipótesis de eficiencia salarial, un ejemplo de estos trabajos es el realizado por Rana, Baten y Kamil (2010), ellos usan un panel de datos no balanceado para la industria manufacturera de comida de Bangladesh, y encuentran que la hipótesis de salario eficiente no es válida. Otro trabajo dentro de esta vertiente es el de Ferdushi, Baten, Kamil y Mustafa (2011), ellos usan un panel de datos no balanceado con una distribución normal truncada para la industria manufacturera de Bangladesh, y encuentran que el salario es uno de los factores significativos que contribuyen a la producción y a la eficiencia técnica. Por último, Karim, Chan y Hassan (2016) analizan el efecto de la política de salario sobre la eficiencia técnica del país en el caso de Malasia. Para alcanzar dicho objetivo, en la primera etapa, se estima la eficiencia técnica para todos los países de la muestra, usando el método no paramétrico. Y en la segunda, 
después de obtener los resultados de la eficiencia técnica, se realiza la estimación de un modelo de Tobit con efectos fijos, donde la eficiencia técnica es la variable dependiente y la variable independiente es el salario mínimo. Se demuestra que no hay diferencia en la eficiencia técnica entre países con y sin política de salario mínimo.

\section{Marco teórico}

Este trabajo se apoya en la hipótesis del salario eficiente y, concretamente, en el modelo de Choi. En el trabajo de Choi (1995), se propone una modificación del modelo de Goodwin (1967) para incorporar la hipótesis del salario eficiente, donde la formulación de la hipótesis del salario eficiente es realizada de acuerdo con el trabajo de Weiss (1990).

En el trabajo de Goodwin (1967), se considera una economía cerrada, no monetaria, en tiempo continuo, donde se produce un solo bien homogéneo que puede ser consumido o acumulado. Existen hogares que participan en el proceso de producción como trabajadores, y obtienen un ingreso por su trabajo, el cual es su único ingreso. La fuerza de trabajo $N$ crece a una tasa exógena constante $n$. Cuando los hogares tienen empleo reciben un salario real $W$ y gastan todo su ingreso $W L$ en consumo, y $L$ es el nivel de empleo. Los emprendedores combinan capital y trabajo en proporciones fijas para producir el bien. La proporción producto capital $\frac{Y}{K}$ está dada por una constante $\sigma$ y la productividad del trabajo $\frac{Y}{L}$ depende del nivel del salario real: $e \equiv \frac{Y}{L}=e(W)$, donde $e$ es el esfuerzo en el trabajo, el cual se asume como continuamente diferenciable. Hay que notar que $\frac{\dot{e}(W)}{e(W)}=\epsilon(W) \frac{\dot{W}}{W}$, donde $\epsilon \equiv \frac{d e(W)}{d W} \frac{W}{e(W)}$ es la elasticidad del esfuerzo del trabajo con respecto al salario real $^{2}$.

La tecnología de producción, por lo tanto, se puede escribir como sigue:

$Y=\operatorname{Min}(e(W) L, \sigma K)$

Todas las ganancias $Y-W L$ se ahorran, se invierten y se operan como capital. Por lo tanto, el ahorro es igual a la inversión. Sin embargo, aunque no hay capital sin utilizar, puede existir desempleo con una tecnología de producción con coeficientes fijos. La siguiente ecuación diferencial se puede interpretar como la solución a la negociación entre trabajadores y emprendedores.

\footnotetext{
${ }^{2} \dot{x}$ es la derivada con respecto al tiempo de la variable $x$
} 
$\frac{\dot{W}}{W}=f(V)=-\gamma+\rho V$

Donde $V$ es la tasa de empleo. Se analiza una aproximación lineal de la solución a la negociación, donde $\gamma$ y $\rho$ son constantes y positivos. Los parámetros $\gamma$ y $\rho$ del mercado de trabajo dependen de la situación institucional especifica, bajo los cuales los contratos son negociados. Dada la tasa de salario real, las firmas escogen el nivel de empleo y los trabajadores escogen su nivel de esfuerzo. La condición de equilibrio del mercado de bienes se expresa como:

$\dot{K}=Y-W L$

Se usa esta condición para expresar las dinámicas de la tasa de empleo $V$ como una función de la tasa real de salario $W$, esto es:

$\frac{\dot{V}}{V}=f(V, W)=(\sigma-n)-u(W) \sigma-\epsilon(W) \frac{\dot{W}}{W}$

Donde $u(W) \equiv \frac{W}{e(W)}$ es la participación del salario en el producto.

\section{Especificación del modelo}

Para cumplir los objetivos planteados anteriormente, se utiliza el enfoque de Frontera de Producción Estocástica para panel de datos propuesto por Battese y Coelli (1995), el cual consiste en estimar un modelo que incluya una frontera de producción, y tome en cuenta una ecuación en la cual las ineficiencias estén especificadas como una función de las variables explicativas. El modelo a estimar se expresa, de la siguiente forma:

$$
\ln Y_{i t}=\beta_{0}+\beta_{i} \ln x_{i t}+v_{i t}-u_{i t}
$$

Donde los índices $i$ son países y $t$ años de observación, $Y$ denota la producción, $x$ es un vector $(1 \mathrm{x} \mathrm{k})$ de funciones conocidas de insumos de producción y otras variables asociadas, $\beta$ es un vector $(\mathrm{k}$ x 1$)$ de parámetros a ser estimados, $v_{i t}$ es la perturbación aleatoria que se asume se distribuye idéntica e independientemente $N\left(0, \sigma_{v}^{2}\right)$. Definimos a $u_{i t}$ como la variable aleatoria, asociada con la ineficiencia técnica de la producción en el modelo de frontera de producción estocástica (5), el cual se distribuye de manera independiente y se expresa, de la siguiente forma:

$$
u_{i t}=\delta Z_{i t}+W_{i t}
$$


Donde $i$ son los países y $t$ años de observación. $Z_{i t}$ es un vector ( $\mathrm{p}$ x 1 ) de variables explicativas; $\delta$, un vector de parámetros a ser estimados; $W_{i t}$, una variable aleatoria que está definida por el truncamiento de la distribución normal con media cero y varianza $\sigma^{2}$.

Por otro lado, el supuesto de que los $u_{i t} s$ y los $v_{i t} s$ están distribuidos independientemente para todo $t=1,2, \ldots, T$ e $i=1,2, \ldots, N$ es una condición que ayuda a simplificar aunque también es restrictiva. Por lo que se requieren modelos alternativos, para considerar la correlación entre las estructuras de los efectos de la ineficiencia técnica y los errores aleatorios en la frontera. Por lo que Battese y Coelli (1995) consideran el método de máxima verosimilitud para la estimación simultánea de los parámetros de la frontera estocástica, y el modelo para las ineficiencias técnicas. La función de verosimilitud se expresa en términos de la varianza de los parámetros, $\sigma_{S}^{2} \equiv \sigma_{v}^{2}+\sigma^{2}$ y $\gamma=\sigma^{2} / \sigma_{S}^{2}$.

La eficiencia técnica de la producción para los $i$ países, en los $t$ años de observación, es definida por la siguiente ecuación:

$T E_{i t}=\exp \left(-U_{i t}\right)=\exp \left(Z \delta_{i t}-W_{i t}\right)$

La predicción de las eficiencias técnicas está basada en su expectativa condicional.

El siguiente paso — en la estrategia para probar las hipótesis planteadas, en este trabajo- es especificar el modelo de frontera de producción estocástica que se utilizará para evaluar la relación entre aumento del salario y crecimiento económico. Para lo cual, se plantean dos funciones de producción: la primera es una función de producción lineal Cobb-Douglas estándar logarítmica y la segunda, una función de producción Translog.

Se asumen como agentes representativos a los países de la muestra donde el producto está dado por una función de producción Cobb-Douglas. Más, aún, se asume una frontera de producción lineal Cobb-Douglas estándar logarítmica, de la siguiente forma:

$$
\ln Y_{i t}=\beta_{0}+\beta_{1} \ln K_{i t}+\beta_{2} \ln L_{i t}+\beta_{3} \ln W_{i t}+v_{i t}-u_{i t}
$$

Donde $Y_{i t}$ representa la variación anual del PIB, en el país $i$ en la muestra en el tiempo $t ; K_{i t}$ es la formación bruta de capital del país $i$ en el tiempo t; $L_{i t}$ es la variación anual del empleo del país $i$ en el tiempo $t$; y $W_{i t}$, el incremento salarial del país $i$, en el tiempo $t$. El vector $\beta$ representa la elasticidad de la variación anual del PIB con respecto al $i$-ésimo insumo; $v_{i t}$ 
se asume como errores independientes e idénticamente distribuidos al azar, los cuales tienen una distribución normal, con media cero y varianza $\sigma^{2} \mathrm{e}$ independientes de $u_{i t}$; los $u_{i t}$ son variables no negativas aleatorias asociadas con la ineficiencia técnica de la producción.

En el modelo de Goodwin (1967), se establece que $\frac{\dot{W}}{W}=f(V)=-\gamma+\rho$, es el poder de negociación de los trabajadores; para la aplicación empírica, se considera a la variación anual de la inflación en el país $i$ en el tiempo $t, I N F_{i t}$, como el resultado del poder de negociación. Así mismo, en el modelo Goodwin (1967), $\frac{\dot{V}}{V}=f(V, W)=(\sigma-n)-u(W) \sigma-\epsilon(W) \frac{\dot{W}}{W} \quad$ es la tasa de empleo; para la aplicación empírica, se utiliza $1-\frac{\dot{V}}{V}$, que se define como la tasa de desempleo.

Se define a $u_{i t}$ como la ecuación que especifica las ineficiencias del modelo y se expresa de la siguiente forma:

$u_{i t}=\delta_{0}+\delta_{1} I N F_{i t}+\delta_{2} D E S_{i t}+W_{i t}$

Donde $D E S_{i t}$ es el desempleo e $I N F_{i t}$, la variación anual de la inflación en el país $i$ en el tiempo $t$, y $\delta$, el vector que representa los parámetros a ser estimados.

Así mismo, se usa un modelo de frontera de producción estocástica para panel de datos propuesto por Battese y Coelli (1995), en la cual los efectos de ineficiencia se asume que están distribuidos normalmente, con efectos de ineficiencia que varían en el tiempo. Investigar la influencia del salario en la eficiencia es posible, haciendo uso de una función de producción estándar Translog, que es aumentada con la variable salario, y se puede expresar de la siguiente forma:

$$
\begin{aligned}
& \ln Y_{i t}=\beta_{0}+\beta_{1} \ln K_{i t}+\beta_{2} \ln L_{i t}+\beta_{3} \ln W_{i t}+\frac{\beta_{4}}{2}\left(\ln K_{i t}\right)^{2}+\frac{\beta_{5}}{2}\left(\ln L_{i t}\right)^{2}+ \\
& \frac{\beta_{6}}{2}\left(\ln W_{i t}\right)^{2}+\beta_{7} \ln W_{i t} \ln K_{i t}+\beta_{8} \ln W_{i t} \ln L_{i t}+\beta_{9} \ln K_{i t} \ln L_{i t}+v_{i t}- \\
& u_{i t}
\end{aligned}
$$

Definimos a $u_{i t}$ como la ecuación que especifica las ineficiencias del modelo y se expresa de la siguiente forma:

$$
u_{i t}=\delta_{0}+\delta_{1} D E S_{i t}+\delta_{2} I N F_{i t}+W_{i t}
$$

Considerando la especificación de la frontera de producción estocástica dada por las ecuaciones (10) y (11), la eficiencia técnica del país i en el t año se 
define como en Battese y Coelli (1988), Taymaz y Saatci (1997) y Kumbhakar y Lovell (2000):

$T E_{i t}=\exp \left(-U_{i t}\right)=\exp \left(Z \delta_{i t}-W_{i t}\right)$

La eficiencia técnica puede ser predicha usando el programa de computadora Frontier 4.1 (Coelli, 1996) y los estimadores de máxima probabilidad del predictor de la ecuación (7), el cual está basado en su expectativa condicional (Battese y Coelli, 1993).

\section{Datos}

Para efectuar esta investigación, se utilizan los datos de Feenstra, Inklaar y Timmer (2015) para la variable variación anual del empleo. Los datos de la variable incremento salarial provienen de ILO (2012), los cuales están expresados en términos de la moneda local de cada país, por lo que, para fines comparativos, se convirtieron a dólares, usando un promedio anual de la cotización del dólar. Los datos de las variables desempleo, variación anual del PIB y formación bruta de capital provienen del Banco Mundial; los de la variable inflación, provienen del World Economic Outlook del Fondo Monetario Internacional.

Para todas las variables, se considera un período que abarca del año 1995 al año 2011. Los países que se estudian son: Argentina, Brasil, Colombia, Chile, México, Perú y Venezuela. Por lo que se cuenta con 119 observaciones para cada variable. Por otro lado, no se considera el uso de variables de control. Esto se debe a que por el momento no hay otros estudios en la literatura que traten este tema. Aunque se podrían utilizar variables de control de la literatura del crecimiento, en el método de frontera de producción se utilizan principalmente insumos de producción, por lo cual se piensa que no es correcto incluirlas. Además, se emplea el supuesto de que los países son agentes representativos.

Derivado del análisis de los datos, encontramos que en promedio, los siete países de América Latina tienen una tasa de crecimiento del 3.5\%, una tasa de incremento salarial de $4.6 \%$, una tasa de formación bruta de capital de $7.9 \%$ y una variación anual del empleo de $2 \%$. Las variables que miden la ineficiencia usadas en este estudio que, además, de acuerdo con la literatura, los resultados indeseables del aumento salarial, son: variación anual de la inflación y desempleo, las cuales tienen valores de 10.9 y 8.85 . Estos valores, sobre todo el de la variable $I N F$, son de suma importancia, ya que tienen valores muy altos que sobrepasan los valores de las variables variación anual del PIB e incremento salarial. 
Tabla 1

Estadísticas básicas

\begin{tabular}{lcccccc}
\hline & $\begin{array}{c}\text { Unidad } \\
\text { de } \\
\text { medida }\end{array}$ & Abreviatura & Media & $\begin{array}{c}\text { Desviación } \\
\text { Estándar }\end{array}$ & Máximo & Mínimo \\
\hline $\begin{array}{l}\text { Función de } \\
\text { frontera de }\end{array}$ & & & & & & \\
producción & & & & & & \\
\hline $\begin{array}{l}\text { Variación } \\
\text { anual del }\end{array}$ & $\Delta \%$ & $Y$ & 3.5 & 4.3 & 18.2 & -10.9 \\
$\begin{array}{l}\text { PIB } \\
\begin{array}{l}\text { Formación } \\
\text { Bruta de } \\
\text { capital }\end{array}\end{array}$ & $\Delta \%$ & $K$ & 7.9 & 21.9 & 108.2 & -38.6 \\
$\begin{array}{l}\text { Variación } \\
\text { anual del } \\
\text { empleo }\end{array}$ & $\Delta \%$ & $L$ & 2 & 2.6 & 8.3 & -12.2 \\
$\begin{array}{l}\text { Incremento } \\
\text { salarial }\end{array}$ & $\Delta \%$ & $W$ & 4.6 & 24.5 & 42.4 & -209.6 \\
\hline
\end{tabular}

\begin{tabular}{|c|c|c|c|c|c|c|}
\hline \multicolumn{7}{|l|}{$\frac{\text { Ecuación }}{\underline{\text { de }}}$} \\
\hline $\begin{array}{l}\text { Variación } \\
\text { anual de la } \\
\text { inflación }\end{array}$ & $\Delta \%$ & $I N F$ & 10.9 & 14.1 & 99.8 & -1.1 \\
\hline Desempleo & $\%$ & $D E S$ & 8.85 & 4 & 18.8 & 2.5 \\
\hline
\end{tabular}

$\mathrm{n}=119$

Fuente: Elaboración propia.

\section{Resultados}

Los resultados de la estimación de la frontera de producción estocástica Cobb-Douglas se pueden observar en la tabla 2, y que para la variable formación bruta de capital se obtuvo un parámetro positivo de .17, el cual es significativo estadísticamente al $1 \%$; de igual manera, la variable incremento salarial tiene un parámetro positivo ${ }^{3}$, el cual es .03 y significativo al $1 \%$. Por otra parte, para la variable variación anual del empleo se estimó un coeficiente negativo de -.07, el cual no es significativo estadísticamente. Para la ecuación de ineficiencia se usan dos variables, desempleo e inflación,

3 Cabe hacer mención que, aunque se encuentra evidencia de una relación positiva entre salario eficiente y crecimiento económico, la relación salario eficiente-crecimiento económico está afectada por el problema de causalidad, es decir, no se conoce si el aumento del salario es el que conlleva a mayor crecimiento económico, o es el crecimiento económico el que produce salarios más altos. 
ambas significativas al 5\%, desempleo tiene un coeficiente positivo de $3.12 \mathrm{e}$ inflación de .79. Los signos positivos de las variables desempleo e inflación indican, que: un aumento en cualquiera de estas variables repercute en un aumento en la ineficiencia. Se encontró que la formación bruta de capital, junto con el incremento salarial, son dos factores que inciden en la determinación de la variación anual del PIB. La gamma estimada, asociada con la varianza de los efectos de ineficiencia técnica, tiene un valor de .99, el cual es significativo al $1 \%$. Más aún, con respecto a la especificación del término de error, los resultados de la estimación muestran que la especificación de la función de producción Cobb Douglas, sin los efectos de ineficiencia, es rechazada; lo cual implica que los efectos de ineficiencia técnica son significativos. La eficiencia media obtenida es de $55 \%$, esto sugiere que, en promedio, el $45 \%$ de los aumentos en la producción se pierden debido a la ineficiencia.

\section{Tabla 2}

Estimados de Máxima-Verosimilitud de la frontera de producción estocástica Cobb-Douglas

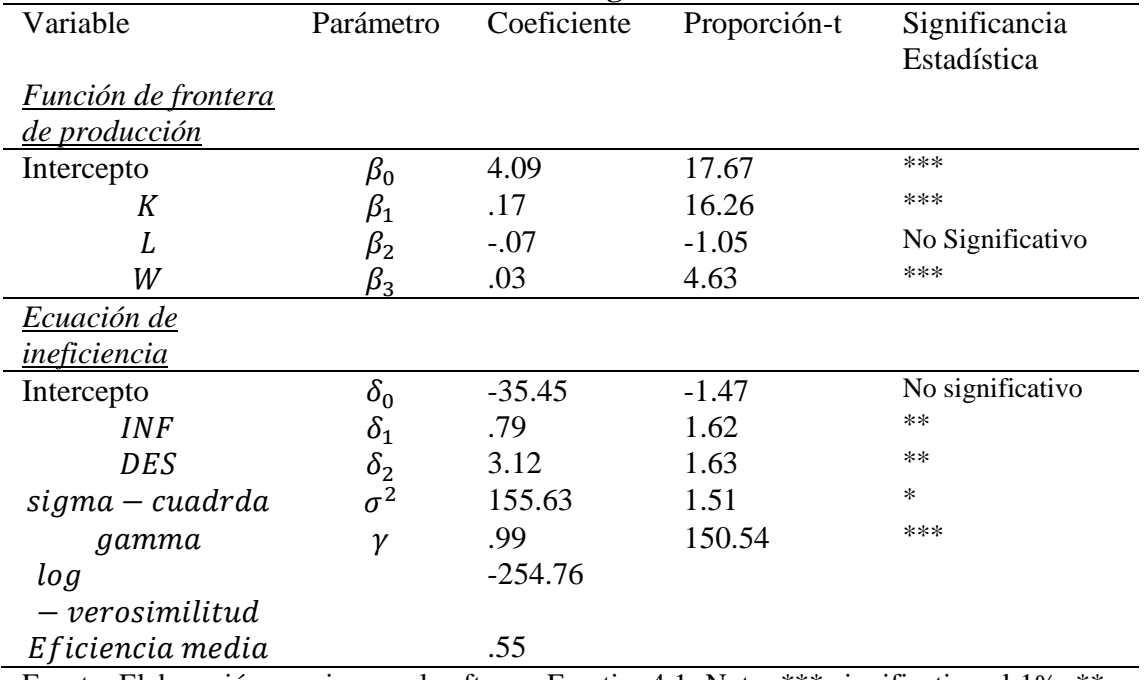

Fuente: Elaboración propia con el software Frontier 4.1. Nota: *** significativo al 1\%, ** significativo al $5 \%$, * significativo al $10 \%$. 
Tabla 3

Estimados de Máxima-Verosimilitud de los parámetros de la frontera de producción estocástica Translog

\begin{tabular}{|c|c|c|c|c|}
\hline Variable & Parámetro & Coeficiente & Proporción-t & $\begin{array}{l}\text { Significancia } \\
\text { Estadística }\end{array}$ \\
\hline \multicolumn{5}{|l|}{ Función de frontera } \\
\hline Intercepto & $\beta_{0}$ & 25.67 & 19.46 & $* * *$ \\
\hline$K$ & $\beta_{1}$ & -28.08 & -12.06 & $* * *$ \\
\hline$L$ & $\beta_{2}$ & 15.19 & 7.25 & $* * *$ \\
\hline$W$ & $\beta_{3}$ & 1.01 & .5 & No significativo \\
\hline$\frac{1}{2} K^{2}$ & $\beta_{4}$ & 13.96 & 12.64 & $* * *$ \\
\hline$\frac{1}{2} L^{2}$ & $\beta_{5}$ & 6.93 & 11.78 & $* * *$ \\
\hline$\frac{1}{2} W^{2}$ & $\beta_{6}$ & 14.22 & 16.52 & $* * *$ \\
\hline$K L$ & $\beta_{7}$ & .43 & 1.35 & No significativo \\
\hline$L W$ & $\beta_{8}$ & -29.44 & -12.52 & $* * *$ \\
\hline$W K$ & $\beta_{9}$ & -.007 & -.009 & No significativo \\
\hline \multicolumn{5}{|l|}{$\begin{array}{ll}\text { Ecuación } & d e \\
\text { ineficiencia } & \\
\end{array}$} \\
\hline Intercepto & $\delta_{0}$ & -31.77 & -9.16 & $* * *$ \\
\hline$I N F$ & $\delta_{1}$ & 2.18 & 5.91 & $* * *$ \\
\hline$D E S$ & $\delta_{2}$ & -3.63 & -5.39 & $* * *$ \\
\hline sigma-cuadrda & $\sigma^{2}$ & 31.51 & 9.76 & $* * *$ \\
\hline gamma & $\gamma$ & .99 & 16088 & $* * *$ \\
\hline $\log$ & & -106.38 & & \\
\hline - verosimilitud & & & & \\
\hline Eficiencia media & & .55 & & \\
\hline
\end{tabular}

Fuente: Elaboración propia con el software Frontier 4.1. Nota: *** significativo al 1\%, ** significativo al $5 \%$, * significativo al $10 \%$.

En la tabla 3, se presentan los resultados de los estimados de máxima verosimilitud de la frontera de producción Translog. La formación bruta de capital tiene un signo negativo, con un valor de -28.08 y es significativa al $1 \%$. Para la variable variación anual del empleo, se obtuvo un coeficiente positivo de 15.19 , el cual es significativo al $1 \%$. La variable incremento salarial tiene un coeficiente positivo de 1.01 , pero no es significativo estadísticamente. Los términos cuadrados de las variables $K, L$ y $W$ todos tienen coeficientes positivos y son significativos al $1 \%$. El valor de los coeficientes de los términos cuadrados son: para la variable $\frac{1}{2} K^{2}, 13.96$; para $\frac{1}{2} L^{2}, 6.93$ y para $\frac{1}{2} W^{2}, 14.22$. Los coeficientes de los términos cruzados $K L$ y $W K$ son estadísticamente no significativos. $K L$ tiene un coeficiente positivo con valor de .43 y $W K$ un coeficiente negativo de -.007. 
Por último, el término cruzado $L W$ es estadísticamente significativo al $1 \%$, su coeficiente es negativo y su valor es -29.44. Los resultados de la estimación de la ecuación de ineficiencias son los siguientes: para la variable inflación se obtuvo un coeficiente de 2.18 , el cual es significativo al $1 \%$ y, para la variable desempleo, el coeficiente es -3.63 , el cual es significativo al $1 \%$. Cabe resaltar que el coeficiente de las variables $K$ y DES no tienen el signo negativo esperado ni significancia estadística del $1 \%$, lo que no distorsiona considerablemente los resultados. El parámetro de varianza, gamma, que alcanza valores entre 0 a 1 , indica que la ineficiencia técnica es estocástica y relevante para obtener una adecuada representación de los datos. El valor proveniente de la estimación es .99 , el cual es significativo al $1 \%$, este resultado indica la presencia de ineficiencias técnicas. El valor de la eficiencia técnica media es de $55 \%$, esto también sugiere que en promedio la ineficiencia técnica es de $45 \%$.

Tabla 4

Eficiencia técnica promedio por país

\begin{tabular}{cc}
\hline País & Eficiencia técnica promedio \\
\hline Argentina & .63 \\
Chile & .61 \\
Perú & .61 \\
Venezuela & .57 \\
México & .54 \\
Colombia & .49 \\
Brasil & .45 \\
\hline
\end{tabular}

Fuente: Elaboración propia con el software Frontier 4.1

La tabla 4 muestra que, en el período de estudio, el país con el nivel promedio más alto de eficiencia técnica es Argentina, con 63\%, seguido por Chile y Perú, ambos con $61 \%$. Mientras que Brasil tiene el nivel más bajo de eficiencia técnica, de los países considerados en este estudio.

En la gráfica 1, se observan los resultados de la eficiencia técnica en promedio para los siete países en consideración. 


\section{Gráfica 1}

Eficiencia técnica promedio anual

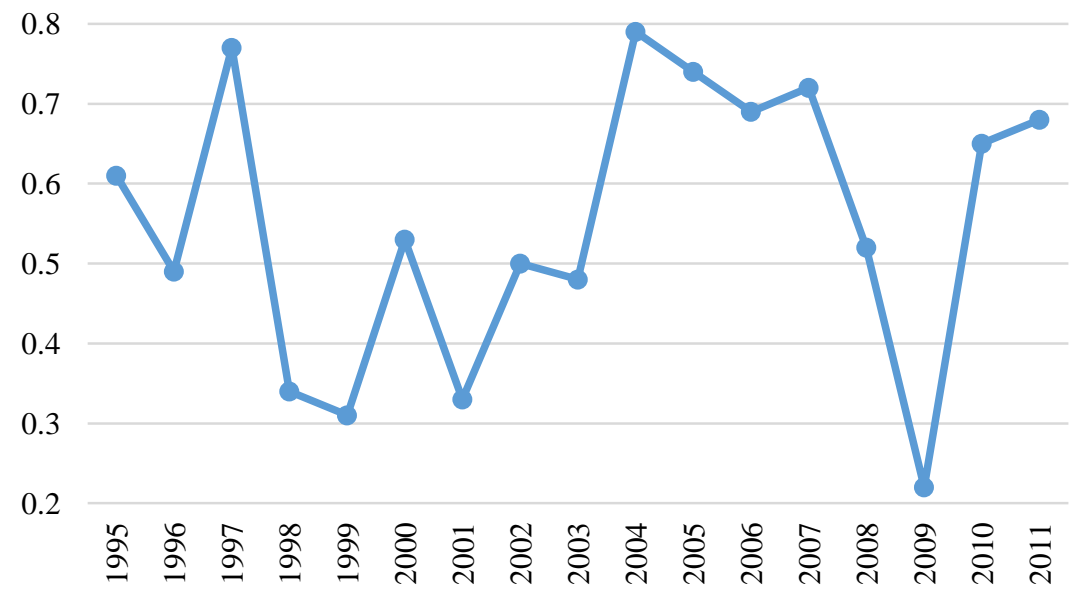

Fuente: Elaboración propia

La gráfica 1 muestra que los años con los valores más altos de eficiencia técnica son 1997 y 2004, ambos años con valores cercanos al 80\%. El año con el valor más bajo es 2009 , con un valor cercano al $20 \%$.

\section{Prueba de hipótesis}

La primera prueba de hipótesis es determinar si la especificación CobbDouglas es una representación adecuada de la función de frontera de producción o no. Esta prueba se hace a través del estadístico de la proporción generalizada de probabilidad, también conocida como prueba LR, que denotaremos con la letra $\lambda$ y está dada por la siguiente ecuación:

$\lambda=-2\left[\ln \left\{L\left(H_{0}\right)\right\}-\ln \left\{L\left(H_{1}\right)\right\}\right]$

Donde $\ln \left\{L\left(H_{0}\right)\right\}$ y $\ln \left\{L\left(H_{1}\right)\right\}$ son los valores de la función de verosimilitud bajo la hipótesis nula y alternativa, respectivamente. La hipótesis nula $H_{0}: \beta_{i j}=0$ es el modelo con restricciones, en este caso, el modelo con la función de producción Cobb-Douglas. La hipótesis alternativa es el modelo con la función de producción Translog. El valor de $\lambda$ tiene una distribución Chi cuadrada y los grados de libertad están dados por el número de restricciones que se le aplican al modelo, en este caso, seis. El valor de la prueba para este caso particular es $\lambda=-2[-254.76-(-106.38)]=$ 296.76, que es mayor que el Chi cuadrado de tablas, al 5\% de significancia, 
que es 12.59 , por lo que rechazamos la hipótesis nula en favor de la función de producción Translog.

Otra prueba importante para determinar si existe la frontera de producción es la que se obtiene a través de la prueba asimétrica de verosimilitud de Coelli (1996). La hipótesis nula es que $\gamma=0$. Dado que la hipótesis alternativa es que $0<\gamma<1$, la prueba tiene una distribución asintótica, los valores críticos los podemos encontrar en Kodde y Palm (1986). Si la hipótesis nula no es rechazada, no se encuentra evidencia de que existe subutilización de la capacidad en los datos, y la frontera de producción es idéntica a la función de producción estándar. De los resultados del modelo obtenemos el valor de la Prueba asimétrica de verosimilitud, el cual es 113.87, este valor debe ser comparado con el valor crítico de la tabla publicada por Kodde y Palm (1986) con 6 grados de libertad, que es 11.911, al $5 \%$ de significancia, y dado que el valor de la prueba es mayor que el valor en la tabla, entonces, rechazamos la hipótesis nula de que no existe evidencia de subutilización de la capacidad de los datos.

Tabla 5

Resultados de las pruebas de hipótesis

\begin{tabular}{lllll}
\hline Hipótesis Nula & $\begin{array}{l}\text { Resultado } \\
\text { Prueba }\end{array}$ & de la & $\begin{array}{l}\text { Valor } \\
\text { Crítico }\end{array}$ & Decisión \\
\hline $\boldsymbol{H}_{\mathbf{0}}: \boldsymbol{\gamma}=\boldsymbol{\beta}_{\mathbf{0}}=\begin{array}{r}\boldsymbol{\beta}_{\mathbf{1}} \ldots \\
=\boldsymbol{\beta}_{\mathbf{6}}\end{array}$ & $\begin{array}{l}296.76 \\
=\mathbf{0}\end{array}$ & 12.59 & Se rechaza $H_{0}$ \\
$\boldsymbol{H}_{\mathbf{0}}: \boldsymbol{\gamma}=\mathbf{0}$ & 113.87 & & & \\
\hline
\end{tabular}

Fuente: Elaboración propia con el software Frontier 4.1

\section{Conclusiones}

En este trabajo, se estudian las implicaciones de la hipótesis de salario eficiente en el nivel macroeconómico, para el caso de siete países de América Latina. Se plantearon dos hipótesis: la primera es que un aumento en el nivel salarial permite alcanzar mayor crecimiento económico y la segunda, que el desempleo y la inflación son variables que aumentan la ineficiencia. Para probar estas hipótesis, se usaron dos tipos de funciones de producción, las cuales son: Cobb-Douglas y Translog, encontrándose que la función de producción Translog es la que mejor caracteriza los datos.

Se descubrió evidencia que apoya la hipótesis de que un aumento en el nivel salarial permite alcanzar mayor crecimiento económico; ya que el coeficiente de la variable incremento salarial es positivo y significativo al $1 \%$, en el modelo con la función de producción Cobb Douglas; mientras que en el modelo con la función de producción Translog, el coeficiente es positivo, 
pero no es estadísticamente significativo. Sin embargo, el término cuadrático de la variable incremento salarial tiene un coeficiente positivo y es estadísticamente significativo al $1 \%$. Pese a que se encuentra evidencia que prueba la relación entre aumento salarial y crecimiento económico, en ambos casos, el coeficiente es muy pequeño con respecto a los coeficientes de las variables $K$ y $L$.

En cuanto a la segunda hipótesis, se encontró evidencia que permite afirmar que las variables inflación y desempleo afectan positivamente a la ineficiencia. Los resultados, cuando se emplea la función de producción Cobb-Douglas, muestran que los coeficientes de las variables $I N F$ y $D E S$ son, respectivamente, .79 y 3.12 , los cuales son significativos al 5\%. Esto significa que ambas variables aumentan la ineficiencia, dado que su signo positivo implica una reducción de la eficiencia. Los resultados del modelo que utiliza la función de producción Translog muestran que la variable desempleo tiene signo negativo, mientras que la variable inflación tiene un coeficiente de correlación de 2.18 y es estadísticamente significativo al $1 \%$. Por tanto, se encuentra que la variable inflación tiene signo positivo, y esto significa que la variable inflación aumenta la ineficiencia.

Así mismo, se probó la existencia de subutilización de los datos y por tanto la existencia de la frontera de producción estocástica. Normalmente, se encuentra que mayores salarios aumentan el esfuerzo de los trabajadores y, por lo tanto, la producción. Pero este estudio solo encuentra evidencia parcial para los siete países de América Latina.

Los resultados de la investigación experimental confirman los resultados obtenidos en los trabajos de Cohn, Fehr, Herrmann y Schneider (2014) y Gerhards y Heinz (2017). Por otro lado, en cuanto a la investigación en el nivel macroeconómico, los resultados muestran evidencia cruzada de la existencia de la relación entre salarios eficientes y crecimiento económico, como sucede en los trabajos de Sabia (2015), Karim, Chan y Hassan (2016) y Tadjoeddin (2016). Por un lado, en el trabajo de Sabia (2015), se encuentra evidencia de la relación entre aumentos salariales y crecimiento en el nivel estatal, para el caso de Estados Unidos. Mientras que Karim, Chan y Hassan (2016) encuentran que aumentos en el salario mínimo tiene un efecto positivo sobre la eficiencia y el crecimiento económico, para el caso de Malasia. En el trabajo de Tadjoeddin (2016), se encuentra evidencia de una relación positiva entre el salario y el crecimiento económico en la industria manufacturera, para el caso de Indonesia.

La presente investigación se puede ampliar siguiendo el trabajo de Sabia (2015) y buscando los sectores donde el aumento en el salario tiene mayor impacto. Por otro lado, los resultados de la eficiencia técnica muestran que 
Argentina es el país con el nivel más alto de eficiencia técnica, mientras que Brasil es el país con el nivel más bajo. Los años con los niveles más altos de eficiencia técnica son 1997 y 2004, mientras que el nivel más bajo de eficiencia técnica se observa en 2009.

\section{Referencias}

[1] Askenazy, P. (2003). "Minimum wage, exports and growth." European Economic Review, 47(1), 147-164.

[2] Battese, G., y Coelli, T. (1988). "Prediction of firm-level technical efficiencies with a generalized frontier production function and panel data." Journal of Econometrics, 38(3), 387-399.

[3] Battese, G. y Coelli, T. (1993). "A Stochastic Frontier Production Function incorporating a model for technical inefficiency effects." Working Paper in Econometrics and Applied Statistics 69/93.

[4] Battese, G. y Coelli, T. (1995). "A model for technical inefficiency effects in a stochastic frontier production function for panel data." Empirical Economics, 20(2), 325-332.

[5] Cahuc, P. y Michel, P. (1996). "Minimum wage unemployment and growth." European Economic Review, 40 (7), 1463-1482.

[6] Choi, H. (1995). "Goodwin's growth cycle and the efficiency wage hypothesis." Journal of Economic Behavior and Organization, 27(2), 223-235.

[7] Coelli, T. J. (1996). "A Guide to FRONTIER Version 4.1: A Computer Program for Stochastic Frontier Production and Cost Function Estimation". CEPA, Working Paper.

[8] Cohn, A., Fehr, E., Herrmann, B. y Schneider, F. (2014). "Social comparison and effort provision: evidence from a field experiment." Journal of the European Economic Association, 12(4), 877-898.

[9] Fanti, L. y Gori, L. (2011). "On economic growth and minimum wages.” Journal of Economics, 103(1), pp.59-82.

[10] Feldstein, M. (2008). "Did wages reflect growth in productivity?" Journal of Policy Modeling, 30(4), 591-594.

[11] Feenstra, R., Inklaar, R. y Timmer, M. (2015). "The Next Generation of the Penn World Table." American Economic Review, 105 (10), 3150-3182,

[12] Ferdushi, K., Baten, A., Kamil, A. y Mustafa, A. (2011). "Wage augmented stochastic frontier model with truncated normal distribution." International Journal of Physical Sciences, 6(14), 3288-3295.

[13] Gerhards, L. y Heinz, M. (2017). "In good times and bad - Reciprocal behavior at the workplace in times of economic crises." Journal of Economic Behavior and Organization, 134, 228-239.

[14] Goodwin, R. M. (1967). A Growth Cycle. Feinstein, C.H. (Ed.), Socialism, Capitalism and Economic Growth (pp. 54-58). Cambridge: Cambridge University Press.

[15] Huang, T. L., Hallam, A., Orazem, P. F. y Paterno, E. M. (1998). "Empirical Tests of Efficiency Wage Models.” Economica, 65(257), 125-143.

[16] Iheanacho, E. (2017). "Emperical Review on the Relationship between Real Wages, Inflation and Labour Productivity in Nigeria. ARDL bounds testing approach.” International Economics and Business, 3(1). 
[17] ILO (2012) Global Wage Report 2012/13: Wages and Equitable Growth. Available in: www.ilo.org

[18] Karim, Z., Chan, S. G. y Hassan, S. (2016). "Minimum Wage Policy and Country's Technical Efficiency." International Journal of Economics and Financial Issues, 6(4), 1729-1735.

[19] Kodde, D. A. y Palm, F. C. (1986). "Wald Criteria for Jointly Testing Equality and Inequality Restrictions". Econometrica, 54(5), 1243.

[20] Kumar, S., Webber, D. J. y Perry, G. (2012). "Real wages, inflation and labour productivity in Australia." Applied Economics, 44(23), 2945-2954.

[21] Kumbhakar, S. y Lovell, C. A. (2000). Stochastic Frontier Analysis, Inglaterra: Cambridge University Press.

[22] Levine, D. (1992). "Can Wage Increases Pay For Themselves? Tests with a Productive Function.” The Economic Journal, 102 (414), 1102-1115.

[23] Lucas, R. (1988). "On the mechanics of economic development." Journal of Monetary Economics, 22 (1), 3-42

[24] Meckl, J. (2001). "Efficiency-wage Unemployment and Economic Welfare in a Model of Endogenous Growth." Labour, 15(4), 579-602.

[25] Mertzanis, H. (2009). "Efficiency Wages, Inflation And Growth." Journal of Economic Development, 34(2), 131-151.

[26] Rana, M., Baten, A. y Kamil, A. (2010). "A stochastic frontier approach for empirical tests of efficiency wage models." Scientific Research and Essays, 5(11), 1234-1242.

[27] Ravn, M. y Sorensen, J. (1999). "Schooling, Training, Growth and Minimum Wages." Scandinavian Journal of Economics, 101, 441-457.

[28] Razzak, W. A. (2015). "Wage, productivity and unemployment: microeconomics theory and macroeconomics data." Applied Economics, 47(58), 6284-6300.

[29] Romer, P. M. (1986). "Increasing Returns and Long-Run Growth." Journal of Political Economy, 94(5), 1002-1037.

[30] Sabia, J. (2015). “Do minimum wages stimulate productivity and growth?”. IZA World of Labor, 221.

[31] Shapiro, C. y Stiglitz, J. (1984). "Equilibrium Unemployment as a Worker Discipline Device." American Economic Review, 74 (3), 433-444.

[32] Solow, R. (1979). "Another possible source of wage stickiness." Journal of Macroeconomics, 1 (1), 79-82.

[33] Stigler, G. (1946). "The Economics of Minimum Wage Legislation." The American Economic Review, 36(3), 358-365.

[34] Tadjoeddin, M. (2016). "Productivity, wages and employment: evidence from the Indonesia's manufacturing sector." Journal of the Asia Pacific Economy, 21 (4), 489-512.

[35] Tang, C. F. (2014). "The effect of real wages and inflation on labour productivity in Malaysia." International Review of Applied Economics, 28(3), 311-322.

[36] Taymaz, E. y Saatci, G. (1997). "Technical Change and Efficiency in Turkish Manufacturing Industries." Journal of Productivity Analysis, 8 (4), 461-475.

[37] Wang, H. y Schmidt, P. (2002). "One-Step and Two-Step Estimation of the Effects of Exogenous Variables on Technical Efficiency Levels". Journal of Productivity Analysis, 18 (2), 129-144.

[38] Wadhwani, S. y Wall, M. (1991). "A Direct Test of the Efficiency Wage Model Using UK Micro-data”. Oxford Economic Papers, 43 (4), 529-548.

[39] Weiss, A. (1990). Efficiency Wages. doi:10.1515/9781400862061 
[40] Xu, S., Huo, L. y Shang, W. (2015). "The Impact of Wage Distributions on Economics Growth Based on Multi-agent Simulation". Procedia Computer Science, 55, 809-817.

[41] Yamaguchi, M. (2017). "Stagnation and minimum wage: Optimal minimum wage policy in macroeconomics". MPRA Paper

[42] Yildirim, Z. (2015). "Relationships among labour productivity, real wages and inflation in Turkey”. Economic Research-Ekonomska Istraživanja, 28(1), 85-103.

[43] Zagler, M. (2011). "Endogenous Growth, Efficiency Wages, and Persistent Unemployment." Review of Economics \& Finance, 1, 34-42. 